\title{
Technology of Local Cross-linking. Part 2: Experimental Results of Local Corneal Cross-linking
}

\author{
${ }^{1}$ Sergey I Anisimov, ${ }^{2}$ Svetlana Y Anisimova, ${ }^{3}$ Anatoliy S Mistryukov, ${ }^{4}$ Natalia S Anisimova \\ ${ }^{5}$ Kirill A Zolotorevskiy, ${ }^{6}$ Ilia A Popov
}

\begin{abstract}
Aim: The aim of the study was to assess the morphological changes in animal corneas following local cross-linking (CXL).

Materials and methods: Experimental comparative study included nine isolated porcine eyeballs (ex vivo group) and nine Chinchilla rabbits, one eye per animal (in vivo group). All the corneas in both groups were saturated with $0.1 \%$ riboflavin and corneal CXL was performed with Locolink device (Transkontakt, Russia), a system that allows to deliver ultraviolet (UV) radiation through a mask of any pattern. One half of each cornea was cross-linked and the other half remained intact. Three corneas of each group were irradiated according to the standard Dresden protocol with $3.0 \mathrm{~mW} / \mathrm{cm}^{2}$ for 30 minutes $(5.4 \mathrm{~J})$; yet, three corneas were irradiated in accelerated CXL mode I with $6.0 \mathrm{~mW} / \mathrm{cm}^{2}$ for 15 minutes $(5.4 \mathrm{~J})$, and yet, three corneas were treated in accelerated CXL mode II with $5.0 \mathrm{~mW} / \mathrm{cm}^{2}$ for 10 minutes $(3.0 \mathrm{~J})$. In the ex vivo group, the corneas of the whole isolated porcine eyeballs were examined with optical coherence tomography (OCT): The stromal demarcation line depth was evaluated by 10 different topographical points per image and the optical density of the corneal layers over the demarcation line was evaluated qualitatively as positive or negative. In the In vivo group, the rabbits were sacrificed 5 days after treatment, and their eyes were enucleated. Corneoscleral buttons were excised and subjected to histological and transmission electron microscopy (TEM) examination.
\end{abstract}

Results: Ex vivo group: Ultrastructural examination and OCT imaging showed that the demarcation line was more superficial after accelerated CXL than after standard CXL. In vivo group. No histological change was detected in the intact area. The re-epithelialization was complete by 72 hours after the procedure. Ultrastructural and histological examination on day 5 following complete re-epithelialization of the cornea showed the presence of bridge-like chains forming cross-links between

${ }^{1}$ Professor, ${ }^{2}$ General Director, ${ }^{3-5}$ Resident, ${ }^{6}$ Ophthalmologist

${ }^{1}$ Department of Ophthalmology, Moscow State University of Medicine and Dentistry named after A.I.Evdakimov, Moscow Russia

${ }^{3}$ Department of Ophthalmology, Moscow State University of Medicine and Dentistry named after A.I.Evdakimov, Moscow Russia; Eye Center "East Sight Recovery", Moscow, Russia

${ }^{4}$ National Medical Research Center, The S. Fyodorov Eye Microsurgery Federal State Institution, Moscow, Russia

2,5,6Eye Center "Vostok-Prozrenie", Moscow, Russia

Corresponding Author: Sergey I Anisimov, Professor Department of Ophthalmology, Moscow State University of Medicine and Dentistry named after A.I.Evdakimov, Moscow Russia, Phone: +79166766601, e-mail: xen3744@yandex.ru collagen fibrils in tangential sections. Keratocytes in irradiated zones were either in the state of apoptosis or were activated and produced newly formed collagen.

Conclusion: The method of local CXL allows to accurately and selectively modify limited regions of the cornea leaving other areas intact. In the case of local irradiation of the cornea, UV structural changes occur only within the irradiated area. The optimal protocol for use in local CXL is the Dresden protocol.

Keywords: Accelerated, Cross-linking, Local, Meta-optics, Personalized.

How to cite this article: Anisimov SI, Anisimova SY, Mistryukov AS, Anisimova NS, Zolotorevskiy KA, Popov IA. Technology of Local Cross-linking. Part 2: Experimental Results of Local Corneal Cross-linking. Int J Kerat Ect Cor Dis 2018;7(1):1-5.

Source of support: Nil

Conflict of interest: None

\section{INTRODUCTION}

Corneal CXL, first developed in the early 2000s, ${ }^{1}$ has since been improved over time and shown to be highly effective in keratoconus patients. ${ }^{2-6}$ Local (personalized) CXL is a modification of the standard CXL protocol that combines the reliability of the standard technology, short rehabilitation time, and the capability of more precise changes in corneal topographic characteristics. ${ }^{2}$ In local CXL, not the whole cornea but its selected areas are irradiated with UV light in order to strengthen its weakest parts.

Local CXL is a novel technology and what changes it causes in the corneal stromal tissue are not yet fully understood. It is known though that after standard CXL procedure, corneal stroma is usually characterized by the presence of so-called demarcation line-the transition area between the cross-linked stromal collagen and intact tissue. Demarcation line can be visualized via OCT examination and is usually located in the middle of stroma of the cornea and is parallel to its surface. Another set of stromal tissue changes consists of structural and ultrastructural changes visible in optical and electron microscopy.

\section{PURPOSE}

To assess the morphological changes in animal corneas following local CXL. 
Table 1: Main parameters of CXL procedure used during CXL procedure in the experimental groups

\begin{tabular}{|c|c|c|c|c|c|c|}
\hline \multicolumn{2}{|c|}{ Numbers of objects involved } & \multirow[b]{2}{*}{ CXL protocol } & \multirow[b]{2}{*}{$\begin{array}{l}\text { Epithelium } \\
\text { status }\end{array}$} & \multirow[b]{2}{*}{$\begin{array}{l}\text { Intensity } \\
(m W)\end{array}$} & \multirow[b]{2}{*}{$\begin{array}{l}\text { Fluence total } \\
\left(\mathrm{J} / \mathrm{cm}^{2}\right)\end{array}$} & \multirow[b]{2}{*}{$\begin{array}{l}\text { UVA irradiation } \\
\text { time (minutes) }\end{array}$} \\
\hline $\begin{array}{l}\text { Ex vivo group } \\
\text { (isolated porcine } \\
\text { eyeballs) }\end{array}$ & $\begin{array}{l}\text { In vivo group } \\
\text { (rabbit eyes) }\end{array}$ & & & & & \\
\hline 3 & 3 & Standard Dresden protocol & Off & 3.0 & 5.4 & 30 \\
\hline 3 & 3 & Accelerated CXL mode I & Off & 6.0 & 5.4 & 15 \\
\hline 3 & 3 & Accelerated CXL mode II & Off & 5.0 & 3.0 & 10 \\
\hline
\end{tabular}

\section{MATERIALS AND METHODS}

The ethical committee of the Eye Center East Sight Recover approved the research. All the experimental studies involving animals and animal tissue were conducted in accordance with Helsinki Declaration.

The current prospective experimental comparative study included two groups of experimental objects: Nine Chinchilla rabbits, one eye involved per animal (in vivo group) and nine isolated porcine eyeballs (ex vivo group).

\section{Both Groups}

Local CXL was performed with the use of the standard Dresden protocol ${ }^{2}$ and two accelerated CXL protocols (Table 1). Briefly, corneal epithelium was debrided and then the cornea was saturated with $0.1 \%$ riboflavin (Pharmstandard, Ufa, Russia) for 30 minutes (Fig. 1). One half of each cornea was irradiated using a Locolink device which allows to deliver UV radiation through a mask of any specified pattern (Transkontakt, Russia). Three corneas of each group were irradiated with the use of the standard Dresden protocol with $3.0 \mathrm{~mW} / \mathrm{cm}^{2}$ for 30 minutes (5.4 J); other three corneas were irradiated in accelerated CXL mode I, ${ }^{7,8}$ with $6.0 \mathrm{~mW} / \mathrm{cm}^{2}$ for 15 minutes $(5.4 \mathrm{~J})$, and yet, three corneas were irradiated in accelerated CXL mode II with $5.0 \mathrm{~mW} / \mathrm{cm}^{2}$ for 10 minutes (3.0 J). During the CXL in isolated porcine eyeballs intraocular pressure of $20 \mathrm{~mm} \mathrm{Hg}$ was maintained with the use of an irrigation system injected via the stump of the optic nerve. In all cases, non-irradiated area of the cornea served as

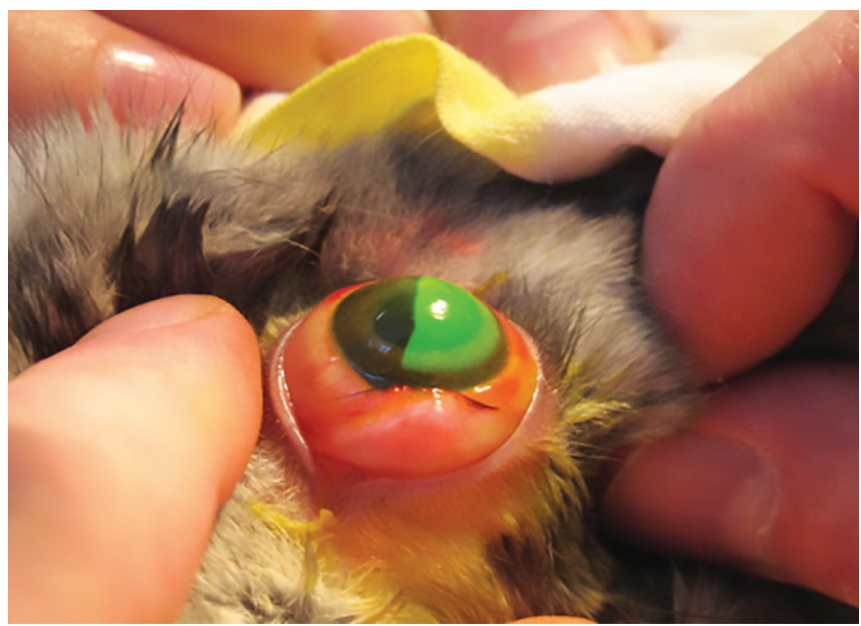

Fig. 1: Irradiation of one half of each rabbit cornea an intact control. Table 1 summarizes main parameters used during CXL procedure in the experimental groups.

\section{Ex vivo Group}

Optical coherence tomography of anterior eye segment was performed in the whole isolated porcine eyeballs. The demarcation line depth was evaluated at 10 different topographical points per image and the optical density of the corneal layers above the demarcation line was assessed qualitatively as positive or negative.

\section{In vivo Group}

After the CXL procedure, all eyes were assessed with biomicroscopy. On day 5 postoperative, the rabbits were sacrificed, and their eyes were enucleated.

Corneoscleral buttons were excised out of the eyeballs and subjected to routine hemotoxylin and eosin staining (HES) tissue section and TEM examination.

\section{RESULTS}

\section{Ex vivo Group}

Ultrastructural examination and OCT imaging (Fig. 2) showed that the depth of the demarcation line was more superficial after accelerated CXL than after standard Dresden protocol. This finding indicates that the absorbed dose following accelerated CXL was higher due to the distribution of UV energy in the smaller volume of tissue.

Table 2 summarizes the main parameters evaluated in the nine porcine eyeballs.

The difference between demarcation line depths in three protocols was statistically significant $(p<0.01$, analysis of variance F-test).

\section{In vivo Group}

During CXL procedure, riboflavin "burnout" was observed in the irradiated zone, but not in the intact zone

Table 2: Demarcation line depth and optical density data evaluated in isolated porcine eyeballs

\begin{tabular}{lll}
\hline & $\begin{array}{l}\text { Demarcation line } \\
\text { CXL protocol }\end{array}$ & Optical \\
depth $(\mu \mathrm{m}) \mathrm{M} \pm \delta$ & density $(+/-)$ \\
\hline Standard Dresden protocol & $290.0 \pm 9.1$ & + \\
Accelerated CXL Mode I & $275.9 \pm 8.0$ & - \\
Accelerated CXL Mode II & $241.3 \pm 9.1$ & + \\
\hline
\end{tabular}



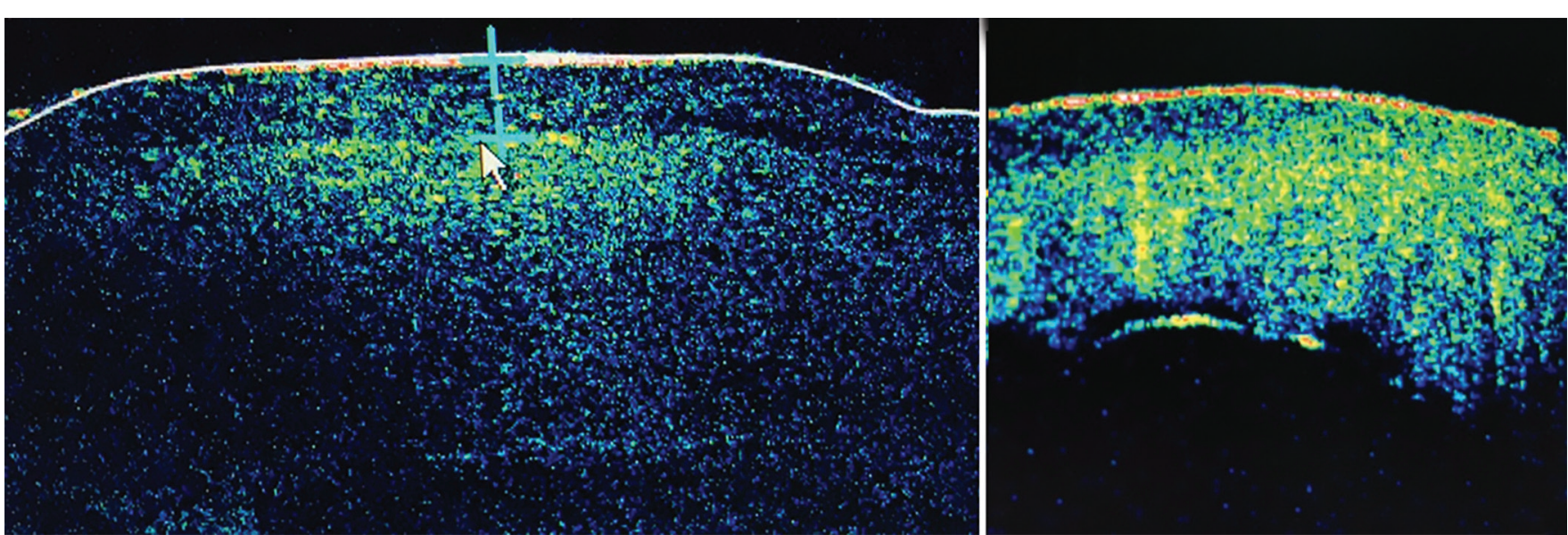

Fig. 2: The OCT and scanned images showing the negative reflecting area of an irradiated cornea. A demarcation line is present at a depth of $273 \mu \mathrm{m}$ following irradiation at a power of $6 \mathrm{~mW} / \mathrm{cm}^{2}$ and an energy of $5.4 \mathrm{~J}$ (left image). Line of demarcation at the border of local irradiation (right image)
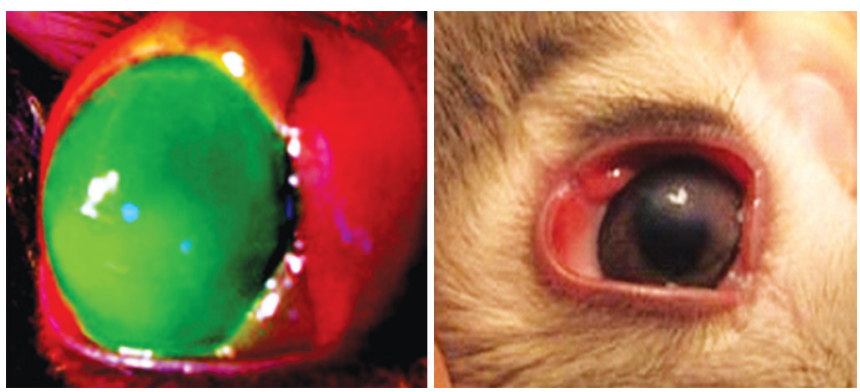

Fig. 3: Images showing a riboflavin "burnout" zone in the upper segment of the cornea after UV irradiation (left image) and a local haze zone in the upper segment of the cornea, corresponding to the UV irradiation zone, following the procedure (right image)

(Fig. 3). Corneal re-epithelialization completed 72 hours postoperative.

No histological change was detected in the intact zone in the HES tissue section examination in all cases. Ultrastructural TEM examination showed that the fibrous structure of collagen in the intact zone was retained (Fig. 4).

Ultrastructural and HES tissue section examination of irradiated areas on day 5 postoperative showed the presence of bridge-like chains that formed cross-links between collagen fibrils in tangential sections (Fig. 5).

Keratocytes in irradiated areas either were in the apoptotic state or were activated and producing newly formed collagen (Fig. 6).

Three spatial rearrangements of collagen fibrils were observed 5 days postoperative: compact fiber bundles (Fig. 7), compact fiber bundles with simultaneous defects of the fibril parallel orientation (Fig. 8), and ring-shaped collagen structures with end-to-end linkages (Fig. 9). The diameter of these structures ranged from 800 to $100 \mathrm{~nm}$ that is comparable to the wavelength range of visible light.

Examination of the rabbit corneas showed that when the radiation power exceeded $3.0 \mathrm{~mW} / \mathrm{cm}^{2}$, even with an energy level remaining lower than $5.4 \mathrm{~J}$, there was an

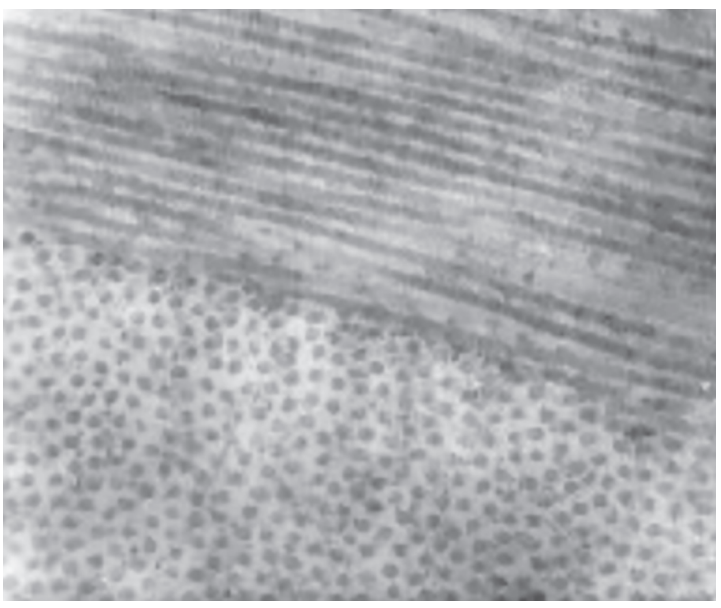

Fig. 4: The TEM image 5 days after removal of epithelium from the intact zone, showing no changes in the size or regular packing of collagen fibers $(\times 16,000)$

increase in the number of twisted collagen fibrils, along with the formation of annular structures.

\section{DISCUSSION}

The morphological findings detected in microscopic examination indicate that higher irradiation power intensified photodestruction processes in cornea during CXL. However, these ring-shaped structures (Fig. 9) may have meta-optic properties, substantially altering the refractive index of the tissue. This was indirectly confirmed by the appearance of a hyporeflecting zone after irradiation of $e x$ vivo porcine corneas with maximum energy (Fig. 2). The presence of these meta-structures in corneas after CXL may explain, at least in part, the discrepancies observed between objective refractive control and the visual acuity in patients. Previously, those discrepancies were often ascribed to the inaccuracy of measuring devices. ${ }^{9}$ The combination of this optical effect with the concept of local CXL may enable the use of these treatment approaches for better control of corneal refraction. 

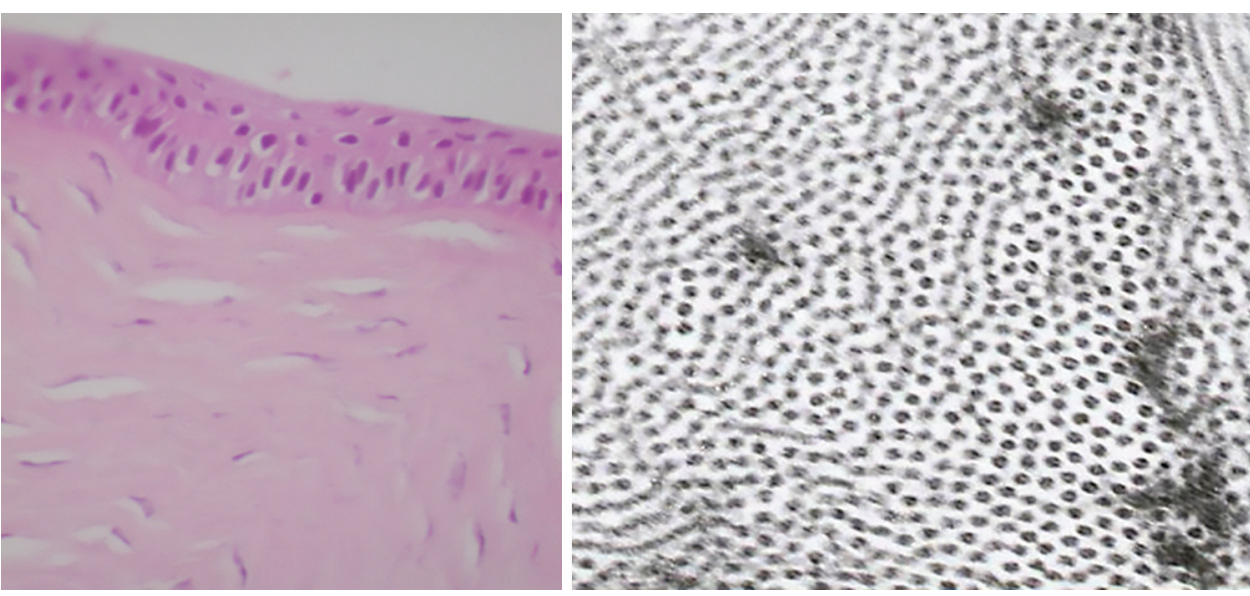

Fig. 5: Evaluation of the irradiated area of the cornea 5 days after the procedure. Histologic examination showing complete re-epithelialization (HES tissue section $\times 240$; left image). TEM examination showing chain-like cross-links between collagen fibers in tangential areas $(\times 18,000$; right image $)$
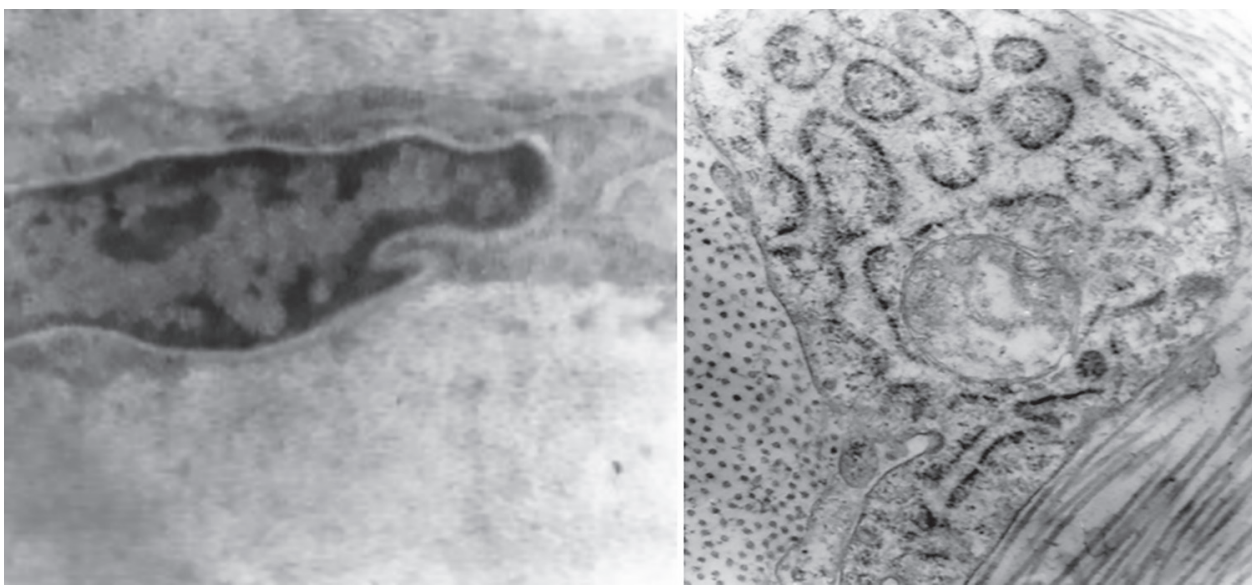

Fig. 6: The TEM images of the irradiated zone showing keratocytes in the state of apoptosis $(\times 8000$, left image $)$ and activated fibroblasts producing collagen $(\times 16,000$, right image $)$

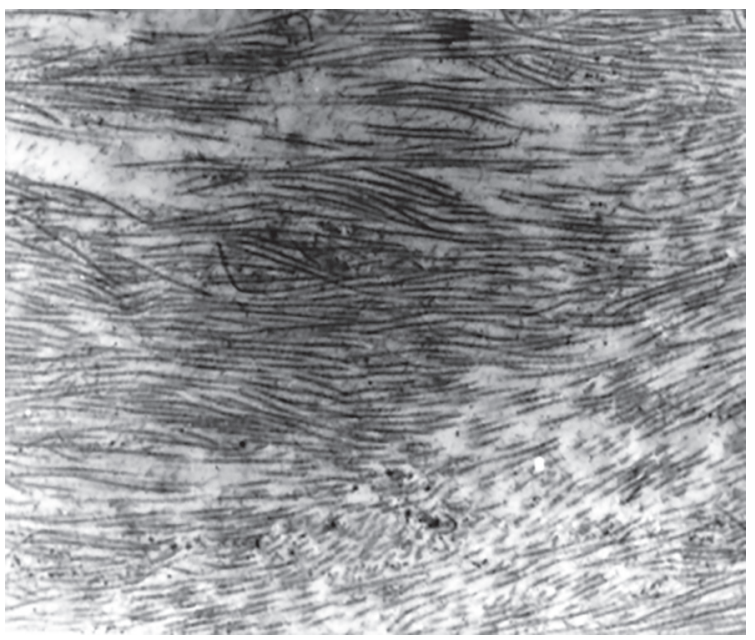

Fig. 7: The TEM image showing compact collagen fibrils 5 days after UV irradiation $(\times 10,000)$

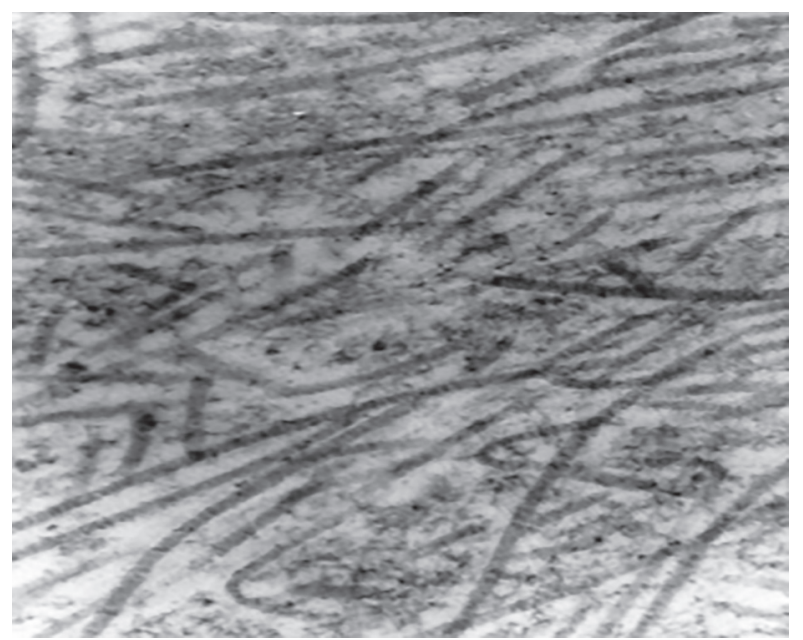

Fig. 8: The TEM image showing compact collagen fibrils with simultaneous defects of the regular fibril orientation 5 days after UV irradiation $(\times 18,000)$ 


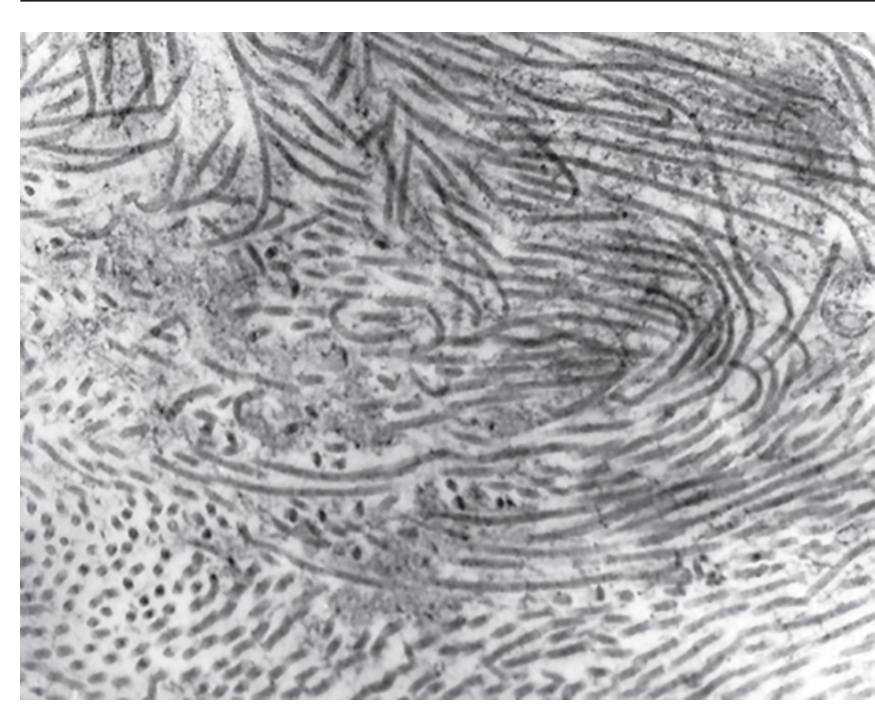

Fig. 9: The TEM image showing the formation of ring-shaped collagen structures in place of regular parallel collagen bundles 5 days after UV irradiation $(\times 16,000)$

\section{CONCLUSION}

The method of local CXL allows to accurately and selectively modify the specified areas of the cornea leaving other areas intact. In the case of local irradiation of the cornea, UV structural changes occur only within the irradiated area. The optimal protocol for use in local CXL is the Dresden protocol.

\section{REFERENCES}

1. Wollensak G, Spoerl E, Seiler T. Riboflavin/ultraviolet-ainduced collagen crosslinking for the treatment of keratoconus. Am J Ophtalmol 2003 May;135(5):620-627.

2. Anisimov SI, Anisimova SY, Mistryukov AS. Personalized (local) UV crosslinking as a treatment of keratoconus and corneal ectasie. Ophtalmology 2017 Jan;15(3):195-199.

3. Spoerl E, Mrochen M, Sliney D, Trokel S, Seiler T. Safety of UVA-riboflavin cross-linking of the cornea. Cornea 2007 May;26(4):385-389

4. Sorkin N, Varssano D. Corneal collagen crosslinking: a systematic review. Issue 1. Ophthalmologica 2014 Apr;232(1):10-27.

5. Raiskup F, Theuring A, Pillunat LE, Spoerl E. Corneal collagen crosslinking with riboflavin and ultraviolet-a light in progressive keratoconus: ten-year results. J Cataract Refract Surg 2015 Jan;41(1):41-46.

6. Alnawaiseh M, Rosentreter A, Böhm MR, Eveslage M, Eter N, Zumhagen L. Accelerated $\left(18 \mathrm{~mW} / \mathrm{cm}^{2}\right)$ corneal collagen cross-linking for progressive keratoconus. Cornea 2015 Nov;34(11):1427-1431.

7. Elbaz U, Shen C, Lichtinger A, Zauberman NA, Goldich $Y$, Chan CC, Slomovic AR, Rootman DS. Accelerated (9-mW/ $\mathrm{cm} 2$ ) corneal collagen crosslinking for keratoconus-a 1-year follow-up. Cornea 2014 Aug;33(8):769-773.

8. Cınar Y, Cingü AK, Türkcü FM, Çınar T, Yüksel H, Özkurt ZG, Çaça I. Comparison of accelerated and conventional corneal collagen cross-linking for progressive keratoconus. Cutan Ocul Toxicol 2014 Sep;33(3):218-222.

9. Greenstein SA, Fry KL, Bhatt J, Hersh PS. Natural history of corneal haze after collagen crosslinking for keratoconus and corneal ectasia: Scheimpflug and biomicroscopic analysis. J Cataract Refract Surg 2010 Dec;36(12):2105-2014. 